\title{
MASK MOUTH: DENTAL GLITCHES BEHIND THE MOUTH MASK IN THE COVID-19 ERA
}

\section{MAURYA $M^{1}$, SAVITHA $S^{2}$, SREESHYLA HS ${ }^{3 *}$, SUSHMA $\mathbf{R}^{\mathbf{1}}$, USHA HEGDE ${ }^{3}$}

1: Department of Public Health Dentistry, JSS Dental College \& Hospital, JSS AHER, Mysuru, India

2: General Dental Practitioner, Bengaluru, India

3: Department of Oral Pathology \& Microbiology, JSS Dental College \& Hospital, JSS

AHER, Mysuru, India

*Corresponding Author: Dr. Sreeshyla HS: E Mail: $\underline{\text { dr.sreeshylahs@jssuni.edu.in }}$

Received $25^{\text {th }}$ June 2021; Revised $28^{\text {th }}$ July 2021; Accepted $29^{\text {th }}$ Aug. 2021; Available online $25^{\text {th }}$ Sept. 2021

https://doi.org/10.31032/IJBPAS/2021/10.9.1050

ABSTRACT

Mouth masks have become a highly essential component in everyone's life. The current pandemic of Covid-19 has forced everybody to compulsorily wear the masks to prevent the danger of acquiring air borne infections. However, its long-term usage has been proposed to cause some ill effects. The oro-facial region that includes the dentition and surrounding structures are one among the primarily affected. It is proposed that the prolonged wearing of mouth mask causes mouth breathing, dehydration, reduced salivary flow and dry mouth. As a consequence, the protective mechanism of saliva is hampered and there is increased bacterial accumulation, which subsequently can lead to halitosis, inflammation of gingiva, dental caries and various infections. This literature review emphasises on "Mask mouth", an entity that has been proposed to address the oral features of major concern occurring due to the long term use of mouth mask.

Keywords: Mouth mask, Mask mouth, Saliva, Dehydration, Mouth breathing, Oral cavity, Covid 19 


\section{INTRODUCTION}

The devastating effect of Covid -19 pandemic has forced changes in the people living all over the world. Personal protection and hygiene have become extremely crucial. In this current pandemic, wearing mask is deliberated as one of the prime protective tools for all, as it has the ability to prevent air borne transmission [1]. Face masks are considered the foremost important step in not only preventing the spread, but also in constraining the spread of the disease [2]. Authorities have made mask wearing a mandate in the public. However, it is proposed that wearing masks for longer time can cause some adverse effects on health $[2,3]$. It may disturb the breathing pattern and can cause increased carbon-dioxide levels producing hypercapnia. Among the vulnerable and Special Group population, lip reading is difficult by people with hearing impairment [2]. Eye discomfort, breathlessness, impaired cognition and acne are considered as the common adverse effects [3].

The other adverse effect proposed is the "Mask mouth", an entity named to indicate all the side effects that are produced in the oral cavity and related structures as a result of long-term wearing of the face mask [36]. It is observed that people with healthy teeth and gums are presenting with newer dental problems $[\mathbf{3}, \mathbf{5}]$. These problems are related to long term wearing of face masks rather than unhygienic oral practices or poor dental hygiene. It is estimated that, almost $50 \%$ of the patients visiting dentists are experiencing signs of Mask Mouth, due to the prolonged use of mouth mask [5, 6]. The name mask mouth has been given after 'meth mouth', a term that describes the dental problems that arise among methamphetamine users usually presenting with cracked, black- and brown-stained teeth [7].

\section{ETIOLOGY AND PATHOPHYSIOLOGY OF MASK MOUTH}

The structure, component, physiology, functions and defensive system of oral cavity is strong enough to resist the diseases that affect the oral cavity. On longterm wearing of the masks, it can alter some of these protective mechanisms in the oral cavity to produce alterations [Table 1] $[3,8]$.

- Covering mouth for long periods of time may increase the dryness of the mouth. Prolonged mask wearers tend to drink less water than usual that may lead to dehydration and dry mouth [3]. Reduced water intake along with dry mouth compounded with the type of food and oral hygiene habits can cause and intensify halitosis [4]. 
Halitosis due to prolonged mask wearing has also been termed as mask breath. Poor oral hygiene and debris on the tongue can also increase the concentration of volatile sulphur compounds in mouth contributing to the halitosis $[7,9,13]$.

- Disturbed breathing patterns have been observed during prolonged mask wearing. Breathing pattern will become more rapid, shallow and mostly done through the mouth, chest, and neck instead of the diaphragm. As breathing through mouth increases inhalation capacity there can be an increased incidence of mouth breathing with the use of a mask [5].

- Saliva plays a crucial role in maintaining oral health by washing away food debris, maintaining an optimal $\mathrm{pH}$ and protecting teeth against cavities $[1,9,10]$. Saliva serves as a protective barrier, as it contains a number of important enzymes, proteins and minerals that aid in fighting against diseases, preventing plaque formation, helping in remineralization and thus keeping the mouth clean $[6,9,11]$. Mouth breathing among extended mask wearers cause surface dehydration, decreased saliva production, reduced salivary flow rate and buffering capacity, there by predisposing to plaque accumulation and increased susceptibility for gingival inflammation and bleeding. It also causes non-flushing of food particles from the teeth and creates a conducive environment for caries forming bacteria and formation of plaque mostly composed of acidforming and acid-tolerable species, such as Streptococcus Mutans and Lactobacilli to thrive upon the sugar in the food particles ultimately producing tooth decay [3-5].

- Mouth breathing, dehydration and decreased saliva causes xerostomia. Dry mouth enhances bacterial build up in the oral cavity leading to halitosis and tooth decay. Increasing bacteria can cause inflammation of the gingiva. Increased bacteria in the mouth and the reduced antibacterial property of saliva add on to the inflammation $[\mathbf{3}, \mathbf{5}, \mathbf{6}]$. Dry mouth also causes difficulty in eating, swallowing and speaking [4, 9]. Mouth breathing, dry mouth and decreased saliva ultimately increases the risk of developing caries, gingivitis, halitosis and 
diseases such as candidiasis and ulcers [3].

- While wearing a mask, there is tendency to trap more carbon dioxide in the mouth than usual. Though this amount of carbon dioxide is low to produce toxicological effects on the body, it can increase the oral microbiome's acidity, which can further increase the risk for infections or inflammatory conditions especially of the gingiva $[\mathbf{1 1}, \mathbf{1 2}]$.

- Dehydration and decreased salivary rate may alter the oral microbiota, increasing the risk for opportunistic infections. Prolonged use of a mask also creates a warm moist environment around the perioral region, which is conducive for bacterial growth. Infections such as candidiasis, angular chelitis and even ulcers can easily occur under such conditions [3].

- When mask is worn for long duration, there is possibility of breathing in one's own exhaled air. During this process there is likelihood of breathing in some of the bacteria that were expelled. This can increase the accumulation of bacteria in the mouth [8].
- The unhygienic use of mouth mask can cause bacterial accumulation on the mask. This can be harmful, as it might trigger or aggravate preexisting oral disease or infection [2].

- Production of moist heat by wearing of masks adjoined by the weather conditions may enhance space infections [2].

- While there is a decreased tendency for drinking water, there is also an increased consumption of coffee and alcohol since the pandemic. Increased use of such acidic drinks has a negative impact on oral health by causing dehydration, staining of the teeth, sensitivity and enamel erosion $[7,9]$.

- Tooth sensitivity can be exacerbated by prolonged periods of mask wearing [9].

- Skin around perioral region may show reddening, irritation and cracks and these effects are termed as maskne [9].

\section{PREVENTION}

Since wearing masks is highly essential and unavoidable, following some precautions while wearing masks can prevent occurrence of mask mouth.

- Adequate protocols should be followed while wearing the masks. 
Clean masks should be worn and should be replaced regularly to prevent microbial contamination [3,

\section{$14,15]$.}

- One of the effective ways of handling is to reduce the amount of time of wearing a mask. Superfluous gatherings should be avoided and working from home if possible should be emphasized. If possible frequent work breaks can be taken or isolated work stations can be created to allow for a shorter duration of mask use. Wearing face masks only when strictly necessary may reduce these undesired side effects $[3,14,15]$.

- Rehydrating frequently with water is advised. It keeps the oral cavity moist, will stimulate salivary secretion there by preventing dry mouth and associated conditions. Simultaneously sugary and acidic drinks should be avoided [1].

- Maintaining good oral hygiene through appropriate brushing technique with the use of proper brushing aids is highly recommended. Flossing interdental areas and a good maintenance of soft tissue hygiene also adds to the prevention [1].

- Use of mouth wash, preferably alcohol free can reduce the microbial load and further prevent the formation of plaque and caries. Chewing sugar-free gum can also help remove food debris [7].

- Preventive dental procedures such as fluoride application, pit and fissure sealants and oral prophylaxis can be undertaken in susceptible patients [3].

- Eat healthy balanced and nutritious diet and avoid sugary and processed food between meals $[\mathbf{7 , 8}$ ].

- Avoid breathing through the mouth [6].

- Educating the public in this regard and regular check on oral health by the person is advised. 
Table 1: Etiology and Pathophysiology of Mask Mouth

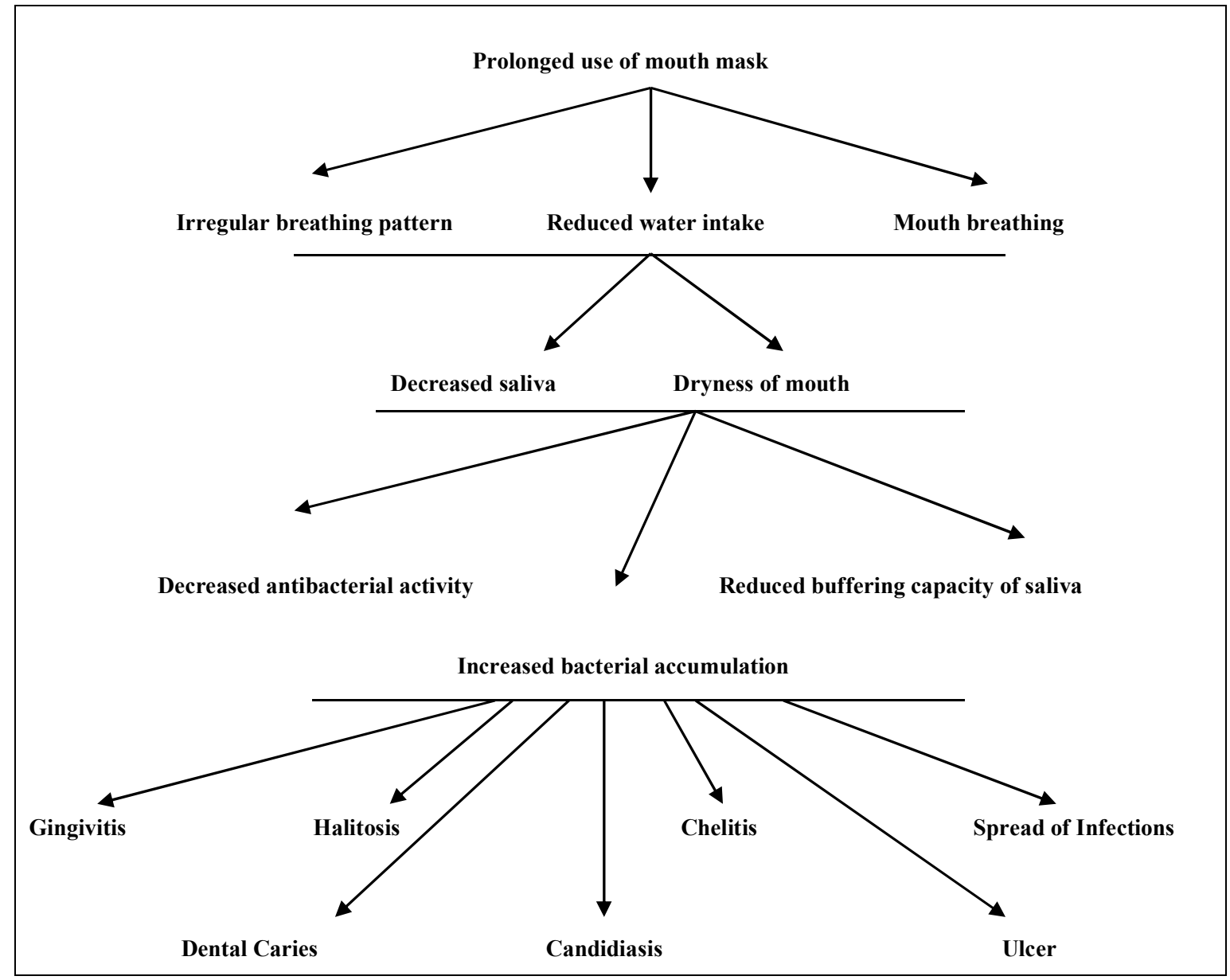

Table 2: Clinical Manifestations of Mask Mouth

\begin{tabular}{|c|}
\hline $\begin{aligned} & \text { Dry mouth } \\
& \bullet \quad \text { Halitosis } \\
\bullet & \text { Gingivitis } \\
\bullet & \text { Dental Caries } \\
\bullet & \text { Candidiasis } \\
& \text { Angular Chelitis } \\
& \bullet \quad \text { Ulcer } \\
& \bullet \quad \text { Acne } \\
& \text { Perioral skin irritation, cracking } \\
& \text { Infections }\end{aligned}$ \\
\hline
\end{tabular}

Table 3: Prevention of Mask Mouth

- Avoid unnecessary wearing of masks

- Maintain mask etiquette

- Frequent rehydration

- Maintain good oral hygiene

- Use of mouthwash and chewing sugar free gums

- Preventive dental procedures

- Healthy eating habits

- Public awareness

- Seeking medical assistance when required 


\section{DISCUSSION}

Wearing masks is a common practice among medical professionals and no such adverse effects have been reported before. There is no scientific evidence to show wearing masks lead to mouth breathing. Evidence based research studies may shed more light on this entity.

A cross-sectional questionnaire study on effects of prolonged usage of N95 respirators and surgical face masks among health care workers suggests that prolonged usage of facemasks can lead to a wide spectrum of side effects relating to nose, facial skin and oral cavity. The results from the study recorded difficulty in breathing on exertion in $58.2 \%$, excessive sweating around the mouth in $67.6 \%$, acne in $56 \%$, skin rashes/redness on the face in $39 \%$, dry mouth in $35.30 \%$ and halitosis in $22.40 \%$ [16]. In a cross-sectional study done to analyse the problem awareness in hygiene mask wearers, humidity and breathing difficulty was reported in more than $45 \%$ of males and $50 \%$ of females [17]. Though non-experimental, these studies show some evidence to suggest that, long-term wearing of mask causes breathing difficulty, dry mouth and halitosis.

Use of facemask induces difficulty in breathing on exertion and excessive sweating around the mouth which results in poor adherence and increased risk of susceptibility to infection. There is a decrease in humidification of air beneath the facemask and decrease in transpiration of the skin around the nasal and perioral region [16]. If masks are not replaced regularly or maintained hygienically, pathogens can accumulate on the mask, risking the spread of infection [2].

The normal concentrations of carbon dioxide while not wearing a face mask varies from 500-900 ppm. Study has shown it to increase in the range from 2150 \pm 192 to $2875 \pm 323 \mathrm{ppm}$, during various working conditions while wearing masks. These concentrations have no toxicological effect. However, concentrations between $1,000 \mathrm{ppm}$ and 10,000 ppm can cause undesirable symptoms such as fatigue, headache and loss of concentration. Hence subjects requiring long-term wearing of facemask should be cautious and aware of these problems [12].

\section{CONCLUSION}

Mask Mouth is a new entity proposed to address the oral health issues arising due to long-term wearing of mouth mask. Decreased water intake, mouth breathing and reduced salivary flow resulting out of prolonged mask wearing can increase the susceptibility of a healthy person to develop oral problems such as dry mouth, halitosis, gingival inflammation, dental caries, infections and ulcers. Suitable 
preventive measures can reduce its occurrence. There is no sound scientific evidence and research in the same regard and the data is restricted to only few reports. Hence a strong evidence based research is required to establish this entity.

\section{Acknowledgement - NIL}

\section{REFERENCES}

[1] Li T, Liu Y, Li M, Qian X, Dai SY. Mask or no mask for COVID-19: A public health and market study. PLoS ONE 2020; 15(8): e0237691. doi:10.1371/journal.pone.023769.

[2] Matuschek C, Moll F, Fangerau $\mathrm{H}$, Fischer JC, Zanker $\mathrm{K}$, Griensven M, et al. Face masks: benefits and risks during the COVID-19 crisis. Eur J Med Res 2020; 25: 32 .

[3] Muley P. 'Mask Mouth'- a novel threat to oral health in the COVID era. Available from file:///F:/zsorted/pub/mask\%20mouth/9.html.

[4] What Is Mask Mouth: Causes, Symptoms \& Prevention | Colgate ${ }^{\circledR}$. Available from file:///F:/z-

sorted $/$ pub/mask $\% 20$ mouth $/ 41 \% 20$

MM\%20yes/3.html.

[5] Mask Mouth - Yes, it is a real thing! | The Family Dental Center. Available from file:///F:/zsorted/pub/mask\%20mouth/22.html.
[6] How Face Masks Are Affecting Oral Health | Fine Arts Dentistry. Available from file://F:/zsorted/pub/mask\%20mouth/40no/6.html.

[7] Licea M. Mask mouth' is a seriously stinky side effect of wearing masks. August 5, 2020. file:///F:/z-sorted/ pub/ mask\% 20mouth/ 29.html.

[8] Mask Mouth - Treating Face Mask Bad Breath. Dec 15, 2020. Available from file://F:/zsorted/pub/mask\%20mouth/21.html.

[9] Stone S. How To Treat And Prevent 'Mask Mouth. 10.02.2020. available from file://F:/Zsorted/pub/mask\%20mouth/11.html.

[10] Singh S, Awasthi N, Gupta T. Mouth Breathing-Its Consequences, Diagnosis \& Treatment. Acta Scientific Dental Sciences 2020; 4(5): 32-41.

[11] Mask Mouth - Yes, it is a real thing! | The Family Dental Center. file: ///F:/z-sorted/ pub/ mask\% 20mouth/ 22.html

[12] Geiss O. Effect of Wearing Face Masks on the Carbon Dioxide Concentration in the Breathing Zone. Aerosol and Air Quality Research 2020; 21: https: //doi.org/10.4209/aaqr.2020.07.04 03. 
[13] Kanehira T, Takehara J, Takahashi D, Honda O, Morita M. Prevalence of oral malodor and the relationship with habitual mouth breathing in children. Journal of Clinical Pediatric Dentistry 2004; 28(4)2; 85-288.

[14] Wilkes M. What to Know About Face Masks \& Your Dental Health. Nov 19, 2020. file:///F:/zsorted/pub/mask $\% 20$ mouth $/ 41 \% 2$ 0MM\%20yes/30.html.

[15] What Is 'Mask Mouth' And How You Can You Prevent It?: Pleasant Family Dentistry. file:///F:/zsorted/pub/mask\%20mouth/40no/8.html

[16] Effects of Prolonged Use of Facemask on Healthcare Workers in Tertiary Care Hospital During COVID-19 Pandemic. Purushothaman PK, Priyangha E. Vaidhyswaran R. Indian $\mathrm{J}$ Otolaryngol Head Neck Surg. https://doi.org/10.1007/s12070020-02124-0.

[17] Morishima $\mathrm{M}$, Kishida $\mathrm{K}$, Uozumi T, Kamijo M. Analysis of problem awareness in hygiene mask wearers: Across sectional survey. International journal of affective engineering 2014; 13(1): 11-18. 